\title{
Editorial
}

\section{Nosocomial Candidemia: An Ounce of Prevention Is Better Than a Pound of Cure}

\author{
Daniel J. Diekema, MD; Michael A. Pfaller, MD
}

This issue of Infection Control and Hospital Epidemiology contains an article by Puzniak et al. ${ }^{1}$ entitled "Has the Epidemiology of Nosocomial Candidemia Changed?" There is no short or simple answer to this question. A review of progress since the early 1980s (prior to the dawn of the "fluconazole era") reveals that several advances have been made in the diagnosis and management of candidemia. Modern, automated blood culture methods have improved our ability to detect candidemia, a thoughtful and rigorous effort has led to the development of standardized methods for susceptibility testing of Candida (and to the demonstration of clinical correlation between in vitro test results and outcome), ${ }^{2-4}$ and several new antifungal agents provide equivalent therapeutic results with less toxicity than amphotericin B deoxycholate..$^{5}$ In addition, the introduction of fluconazole prophylaxis in selected high-risk patient populations (eg, patients receiving a bone marrow transplant and high-risk patients receiving a liver transplant) has resulted in decreased infection rates in these groups ${ }^{6,7}$ and may be associated with an overall decline in mortality due to invasive candidiasis. ${ }^{8}$ But in keeping with the maxim that "no good deed goes unpunished," widespread azole use may have facilitated a less encouraging change in the epidemiology of nosocomial candidemia: the emergence of $C$. glabrata as a more frequent nosocomial pathogen. ${ }^{9}$ Although the decreased susceptibility of $C$. glabrata to azoles and amphotericin $B$ makes this development disturbing, the other species commonly causing nosocomial candidemia ( $C$. albicans, $C$. tropicalis, and $C$. parapsilosis) remain susceptible to fluconazole, ${ }^{10}$ and the feared emergence of $C$. kruse $i$ as a more common cause of nosocomial candidemia has not occurred.
What has not changed is that hospital acquisition of Candida bloodstream infection (BSI) remains a devastating complication of healthcare delivery. The study by Puzniak et al. is the latest in a series of recent articles ascribing an extremely high crude (35\% to $61 \%$ ) and attributable (24\% to 49\%) mortality to nosocomial candidemia. ${ }^{1,11-14}$ Acquiring candidemia in the hospital carries no less risk of death during hospitalization today than it did in the 1980s and early 1990 s. ${ }^{15-20}$ Could it be that this unchanging, high crude mortality means that nosocomial candidemia is merely a marker for severe underlying illness, but doesn't itself contribute significantly to mortality? Prospective clinical trials generally estimate attributable mortality to be much lower than do retrospective cohort designs, ${ }^{21,22}$ and we have previously outlined reasons why a retrospective cohort design might overestimate attributable mortality. ${ }^{14}$ However, we believe that estimates based on the presence of Candida in sterile sites within 48 hours of death or at autopsy ${ }^{21,22}$ grossly underestimate attributable mortality by not including deaths among patients who, although they may clear their infection, die of downstream effects of the physiologic insult sustained during infection. There is evidence supporting a substantial contribution of nosocomial candidemia to mortality, including (1) the high attributable mortality estimates from several retrospective cohort studies, ${ }^{13,14,20}$ (2) the independent association of Candida BSI with mortality in large studies using multivariate models to examine microbiologic risk factors for mortality among patients with BSI, ${ }^{23,24}$ and (3) the mortality benefit documented in association with reduced rates of candidiasis in patients receiving a bone marrow transplant with the use of fluconazole prophylaxis. ${ }^{6}$

So will advances in treatment lead to reductions in Candida-associated mortality? As Puzniak et al. point out,

Drs. Diekema and Pfaller are from the Division of Medical Microbiology, Department of Pathology, and Dr. Diekema is also from the Division of Infectious Diseases, Department of Internal Medicine, University of Iowa Carver College of Medicine, Iowa City, Iowa. Dr. Pfaller is also from the Department of Epidemiology, University of Iowa College of Public Health, Iowa City, Iowa.

Address reprint requests to Daniel J. Diekema, MD, C 606 GH, University of Iowa College of Medicine, 200 Hawkins Drive, Iowa City, IA 52242. 
their study and other recent studies predate the wide availability of the new agents caspofungin and voriconazole. Future studies may yet demonstrate that these agents are more efficacious than amphotericin and fluconazole; however, current data demonstrate only equivalent efficacy for caspofungin (although an important reduction in toxicity when compared with amphotericin B deoxycholate ${ }^{21}$ ), and published data comparing voriconazole with available agents for candidemia are not yet available. One study suggests that combination therapy may improve outcome in patients with nosocomial candidemia, but these data are complicated by an imbalance in severity of illness score between study arms and therefore require confirmation and extension to other agents and comparators. ${ }^{25}$

The risk factors for nosocomial candidemia have been well established and, as Puzniak et al. describe, have not changed significantly during the past two to three decades. Presence of a central venous catheter, intensive care unit (ICU) stay, renal failure, surgery, receipt of antibiotics (increasing risk with each additional antibiotic ${ }^{26}$ ), and receipt of total parenteral nutrition are all recognized to increase the risk for nosocomial candidemia.,26-28 One important risk factor that was not examined in the study by Puzniak et al. is preexisting colonization with Candida at other body sites. Intensity of Candida colonization is a wellknown risk factor for subsequent infection, ${ }^{26,28}$ and we suspect that the association they found between gastric acid suppression and candidemia reflects an associated increased risk for gastrointestinal colonization and overgrowth with Candida, previously described in the National Epidemiology of Mycoses Survey. ${ }^{29}$ The gastrointestinal tract may not only serve as a source of candidemia (through translocation, particularly in critically ill patients receiving total parenteral nutrition or chemotherapeutic agents), but may also increase risk by serving as a reservoir for increased colonization density at other body sites.

Although candidemia is usually deemed to arise endogenously (preceded by colonization with the infecting strain), two other studies in this issue of Infection Control and Hospital Epidemiology remind us that Candida species are also transmitted from patient to patient in the healthcare setting. ${ }^{30,31}$ Although this is a well-described phenomenon for $C$. parapsilosis, a species of Candida for which exogenous acquisition from contaminated infusates, the hospital environment, or the hands of healthcare workers is often implicated, ${ }^{29,32,33}$ other Candida species may also be transmitted between patients, probably on the hands of healthcare workers. ${ }^{30,34,35}$ Nor does an "endogenous" source of candidemia exclude in-hospital transmission of Candida as an important factor in infection. Candida species are common colonizers of human hands (particularly subungual spaces ${ }^{36}$ ), so exposure to Candida in the hospital environment is undoubtedly a common event. ${ }^{37}$ Established risk factors (eg, antibiotic and device use) then favor the establishment of colonization and subsequent infection. Understanding this sequence of events has important implications for preventing morbidity and mortality resulting from nosocomial candidemia.
We agree with Puzniak et al. that better prevention methods will decrease candidemia-associated mortality much more than will advances in therapy. In other words, prevention is primary. Prevention of nosocomial candidemia should involve five strategies. First, intensive programs to maximize adherence to current hand hygiene recommendations are essential. Both alcohol and chlorhexidine are effective in killing Candida species on the hands of healthcare workers ${ }^{38}$ and will decrease the risk of patients acquiring Candida colonization and subsequent infection in the healthcare setting. Second, strategies to improve adherence to current recommendations for placement and care of central venous catheters are important. ${ }^{39}$ An educational program emphasizing important components of these guidelines successfully reduced catheterrelated BSIs in an ICU. ${ }^{40}$ Of note, the authors of this study reported 9 Candida BSIs in the 18-month preintervention period ( $12 \%$ of all nosocomial BSIs in their ICU) and not a single episode of catheter-associated nosocomial candidemia during the 18 months after the educational program. ${ }^{40}$ Third, the importance of antibiotic use as a risk factor for nosocomial candidemia suggests that control of antimicrobial use is an important component of candidemia prevention. These three strategies-improved hand hygiene, optimal catheter placement and care, and prudent antimicrobial use-should form the bedrock of our approach to prevent morbidity and mortality resulting from nosocomial candidemia. Of secondary importance are the uses of presumptive (empiric) and prophylactic antifungal agents to decrease morbidity and mortality resulting from nosocomial candidemia.

Early empiric antifungal therapy should be guided by an understanding of the most important risk factors for nosocomial candidemia. The ICU patient with a central venous catheter, heavy antimicrobial exposure, a fever without a clear source, and Candida colonization at any site has a high risk for candidemia and may benefit from early empiric therapy. ${ }^{5}$ Further study should be undertaken to more precisely define risk-a generalizable "candidemia score" that can be applied in an ICU environment to assist in making decisions about empiric antifungal therapy.

Decisions about expanding prophylactic antifungal use to the non-neutropenic ICU patient are substantially more difficult. Although well-designed, placebo-controlled trials have demonstrated a reduction in invasive candidiasis among surgical ICU patients who receive fluconazole prophylaxis, ${ }^{41,42}$ the study populations selected were at high risk for candidemia, and the generalizability of the results has been questioned. ${ }^{43}$ The potentials for drug toxicity, drug interactions, and the emergence of antifungalresistant Candida species are arguments against a blanket recommendation to use prophylactic antifungal agents for non-neutropenic ICU patients. In our view, any approach to prophylaxis in this population must be institution specific and can be justified only if (1) major and concerted efforts have been made to improve hand hygiene, catheter placement and care, and antimicrobial use practices; (2) the rate of nosocomial candidemia or invasive candidiasis remains 
elevated despite these efforts; and (3) a local observational study can define (using a "prediction rule") a subpopulation within the ICU with a cumulative incidence of invasive candidiasis approaching or exceeding $10 \%$. Using this approach, we suspect that few institutions will find the need to expand antifungal prophylaxis outside of the transplant populations for which it is already recommended. ${ }^{5}$

\section{REFERENCES}

1. Puzniak L, Teutsch S, Powderly W, Polish L. Has the epidemiology of nosocomial candidemia changed? Infect Control Hosp Epidemiol 2004;25: 628-633.

2. Rex JH, Pfaller MA. Has antifungal susceptibility testing come of age? Clin Infect Dis 2002;35:982-989.

3. National Committee for Clinical Laboratory Standards. Method for Antifungal Disk Diffusion Susceptibility Testing of Yeasts. Wayne, PA: National Committee for Clinical Laboratory Standards; 2004. Approved guideline M44-A

4. National Committee for Clinical Laboratory Standards. Reference Method for Broth Dilution Antifungal Susceptibility Testing of Yeasts: Approved Standard, ed. 2. Wayne, PA: National Committee for Clinical Laboratory Standards; 2002. Document M27-A2.

5. Pappas PG, Rex JH, Sobel JD, et al. Guidelines for treatment of candidiasis. Clin Infect Dis 2004;38:161-189.

6. Marr KA, Seidel K, Slavin MA, et al. Prolonged fluconazole prophylaxis is associated with persistent protection against candidiasis-related death in allogeneic marrow transplant recipients: long-term follow-up of a randomized, placebo-controlled trial. Blood 2000;96:2055-2061.

7. Winston DJ, Pakrasi A, Busuttil RW. Prophylactic fluconazole in liver transplant recipients: a randomized, double-blind, placebo-controlled trial. Ann Intern Med 1999;131:729-737.

8. McNeil MM, Nash SL, Hajjeh RA, et al. Trends in mortality due to invasive mycotic diseases in the United States, 1980-1997. Clin Infect Dis 2001;33:641-647.

9. Trick WE, Fridkin SK, Edwards JR, Hajjeh RA, Gaynes RP, the NNIS System hospitals. Secular trend of hospital acquired candidemia among intensive care unit patients in the U.S. during 1989-1999. Clin Infect Dis 2002;35:627-630.

10. Pfaller MA, Messer SA, Hollis RJ, Jones RN, Diekema DJ. In vitro activities of ravuconazole and voriconazole compared with those of four licensed systemic antifungal agents against 6,970 clinical isolates of Candida spp. Antimicrob Agents Chemother 2002;46:1723-1727.

11. Diekema DJ, Beekmann SE, Chapin K, Morel K, Munson E, Doern GV. Epidemiology and outcome of nosocomial and community onset bloodstream infection. J Clin Microbiol 2003;41:3655-3660.

12. Edmond MB, Wallace SE, McClish DK, et al. Nosocomial bloodstream infections in United States hospitals: a three years' analysis. Clin Infect Dis 1999;29:239-244.

13. Gottfredsson M, Vredenburgh JJ, Xu J, Schell WA, Perfect JR Candidemia in women with breast carcinoma treated with high-dose chemotherapy and autologous bone marrow transplant. Cancer 2003;98:24-30.

14. Gudlaugsson O, Gillespie S, Lee $\mathrm{K}$, et al. Attributable mortality of nosocomial candidemia, revisited. Clin Infect Dis 2003;37:1172-1177.

15. Fraser VJ, Jones M, Dunkel J, Storfer S, Medoff G, Dunagan WC. Candidemia in a tertiary care hospital: epidemiology, risk factors, and predictors of mortality. Clin Infect Dis 1992;15:414-421.

16. Harvey RL, Myers JP. Nosocomial fungemia in a large community teaching hospital. Arch Intern Med 1987;147:2117-2120.

17. Horn R, Wong B, Kiehn TE, Armstrong D. Fungemia in a cancer hospital: changing frequency, earlier onset, and results of therapy. Rev Infect Dis 1985;7:646-655.

18. Klein JJ, Watanakunakorn C. Hospital-acquired fungemia: its natural course and clinical significance. Am J Med 1979;67:51-58.

19. Marsh PK, Tally FP, Kellum J, Callow A, Gorbach SL. Candida infections in surgical patients. Ann Surg 1983;198:42-47.

20. Wey SB, Mori M, Pfaller MA, Woolson RF, Wenzel RP. Hospital acquired candidemia: attributable mortality and excess length of stay. Arch Intern Med 1988;148:2642-2645.
21. Mora-Duarte J, Betts $\mathrm{R}$, Rotstein $\mathrm{C}$, et al. Comparison of caspofungin and amphotericin B for invasive candidiasis. $N$ Engl J Med 2002;347: 2020-2029.

22. Rex JH, Bennett JE, Sugar AM, et al. A randomized trial comparing fluconazole with amphotericin B for the treatment of candidemia in patients without neutropenia. N Engl J Med 1994;331:1325-1330.

23. Pittet D, Li N, Woolson RF, Wenzel RP. Microbiological factors influencing outcome of nosocomial bloodstream infection: a 6-year validated, population-based model. Clin Infect Dis 1997;24:1068-1078.

24. Weinstein MP, Towns ML, Quartey SM, et al. The clinical significance of positive blood cultures in the 1990s: a prospective comprehensive evaluation of the microbiology, epidemiology, and outcome of bacteremia and fungemia in adults. Clin Infect Dis 1997;24:584-602.

25. Rex JH, Pappas PG, Karchmer AW, et al. A randomized and blinded multicenter trial of high-dose fluconazole plus placebo versus fluconazole plus amphotericin B as therapy for candidemia and its consequences in nonneutropenic subjects. Clin Infect Dis 2003;36:1221-1228.

26. Wey SB, Mori M, Pfaller MA, Woolson RF, Wenzel RP. Risk factors for hospital-acquired candidemia: a matched case-control study. Arch Intern Med 1989;149:2349-2353.

27. Blumberg HM, Jarvis WR, Soucie JM, et al. Risk factors for candidal bloodstream infections in surgical intensive care unit patients: the NEMIS prospective multicenter study. Clin Infect Dis 2001;33:177-186.

28. Pittet D, Monod M, Suter PM, Frenk E, Auckenthaler R. Candida colonization and subsequent infections in critically ill surgical patients. $A n n$ Surg 1994;220:751-758.

29. Saiman L, Ludington E, Dawson JD, et al. Risk factors for Candida species colonization of neonatal intensive care unit patients. Pediatr Infect Dis J 2001;20:1119-1124.

30. Shin JH, Kim M-N, Shin DH, et al. Genetic relatedness among Candida tropicalis isolates from sporadic cases of fungemia in two university hospitals in Korea. Infect Control Hosp Epidemiol 2004;25:634-640.

31. Posteraro B, Bruno S, Boccia S, et al. Candida parapsilosis bloodstream infection in pediatric oncology patients: results of an epidemiologic investigation. Infect Control Hosp Epidemiol 2004;25:641-645.

32. Diekema DI, Messer SA. Hollis RI, Wenzel RP, Pfaller MA. An outbreak of Candida parapsilosis endocarditis. Diagn Microbiol Infect Dis 1997;29: 147-153.

33. Sanchez V, Vazquez JA, Barth-Jones D, Dembry L, Sobel JD. Nosocomial acquisition of Candida parapsilosis: an epidemiologic study. Am J Med 1993:94:577-582.

34. Doebbeling BN, Hollis RJ, Isenberg HD, Wenzel RP, Pfaller MA Restriction fragment analysis of a Candida tropicalis outbreak of sternal wound infections. J Clin Microbiol 1991;29:1268-1270.

35. Marco F, Lockhart SR, Pfaller MA, et al. Elucidating the origins of nosocomial infections with Candida albicans by DNA fingerprinting with the complex probe Ca3. I Clin Microbiol 1999;37:2817-2828.

36. McGinley KJ, Larson EL, Leyden JJ. Composition and density of microflora in the subungual space of the hand. I Clin Microbiol 1988;26:950953.

37. Strausbaugh LJ, Sewell DL, Ward TT, Pfaller MA, Heitzman T, Tjoelker R. High frequency of yeast carriage on hands of hospital personnel. $J$ Clin Microbiol 1994;32:2299-2300.

38. Boyce JM, Pittet D. Guideline for hand hygiene in health-care settings. MMWR 2002;51(RR-16):1-45

39. O'Grady NP, Alexander M, Dellinger EP, et al. Guidelines for the prevention of intravascular catheter-related infections. MMWR 2002;51(RR10):1-29.

40. Coopersmith CM, Rebmann TL, Zack JE, et al. Effect of an education program on decreasing catheter-related bloodstream infections in the surgical intensive care unit. Crit Care Med 2002;30:59-64.

41. Garbino J, Lew DP, Romand JA, Hugonnet S, Auckenthaler R, Pittet D. Prevention of severe Candida infections in nonneutropenic, high-risk critically-ill patients: a randomized, double-blind, placebo-controlled trial in patients treated by selective digestive decontamination. Intensive Care Med 2002;28:1708-1717.

42. Pelz RK, Hendrix CW, Swoboda SM, et al. Double-blind placebo-controlled trial of fluconazole to prevent candidal infections in critically ill surgical patients. Ann Surg 2001;233:542-548.

43. Sobel JD, Rex JH. Invasive candidiasis: turning risk into a practical prevention policy? Clin Infect Dis 2001;33:187-190. 